Aim of the study: To use different beam arrangements and numbers to plan intensity-modulated radiation therapy (IMRT) and investigate their effects on low and high radiation doses delivered to the functional lung, in order to reduce radiation-induced lung damage.

Material and methods: Ten patients with stage I-III non-small cell lung carcinoma (NSCLC) underwent IMRT. Beam arrangements were selected on the basis of orientation and dose-volume histograms to create SPECT-guided IMRT plans that spared the functional lung and maintained target coverage. Four different plans, including CT-7, SPECT-7, SPECT-4, SPECT-5 with different beam arrangements, were used. The differences of conformity index $(\mathrm{Cl})$, heterogeneity index $(\mathrm{HI})$ between the plans were analyzed, by using a paired t-test. Results: The seven-beam SPECT (SPECT-7) plan reduced the volume of the functional lung irradiated with at least 20 Gy (FV20) and 30 Gy (FV30) by $26.02 \% \pm 15.45 \%$ and $14.41 \% \pm 16.66 \%$, respectively, as compared to the seven-beam computed tomography (CT-7) plan. The $\mathrm{Cl}$ significantly differed between the SPECT-7 and SPECT-4 plans and between the SPECT- 5 and SPECT- 4 plans, but not between the SPECT-5 and SPECT-7 plans. The Cls in the SPECT-5 and SPECT-7 plans were better than that in the SPECT-4 plan. The heterogeneity index significantly differed among the three SPECT plans and was best in the SPECT-7 plan.

Conclusions: The incorporation of SPECT images into IMRT planning for NSCLC greatly affected beam angles and number of beams. Fewer beams and modified beam angles achieved similar or better IMRT quality. The lowdose volumes were lower in SPECT-4.

Key words: non-small lung carcinoma, intensity-modulated radiation therapy, dosiometry beam angle, number of beams.

Contemp Oncol (Pozn) 2014; 18 (6): 436-441 DOI: $10.5114 /$ wo.2014.46237

\section{Impact of different beam directions on intensity-modulated radiation therapy dose delivered to functioning lung tissue identified using single-photon emission computed tomography}

\author{
Qin Tian ${ }^{1^{\star}}$, Fucheng Zhang ${ }^{2^{\star}}$, Yanming Wang ${ }^{1}$, Weiqiang $\mathbf{Q} \mathbf{u}^{1}$
}

'Department of Radiotherapy, The General Hospital of Jinan Military Command, Jinan, Shandong Province, China

2Department of Gastroenterology, The General Hospital of Jinan Military Command, Jinan, Shandong Province, China

${ }^{*}$ Qin Tian and Fucheng Zhang contributed equally to this work.

\section{Introduction}

Lung cancer is the leading cause of cancer-related mortality worldwide [1-3]. Although survival rates can be improved by intensifying radiotherapy [4], attempts at dose escalation are limited by radiation damage to the normal lung in the form of radiation pneumonitis. The incidence of pneumonitis depends on the volume of the normal, functioning lung that has received $\geq 20$ Gy of radiation (FV20) and the mean lung dose (MLD), with the risk of pneumonitis being greater than 10\% when FV20 exceeds 30\% [5-7]. Joao Seco et al. found that protons generate larger high-dose regions than photons because of range uncertainties [8]. Single-photon emission computed tomography (SPECT) with Tc-99m macroaggregated albumin can depict the spatial distribution of lung perfusion, which has been shown to be proportional to lung function. This functional information has been used to identify normally functioning lung tissue when planning intensity-modulated radiation therapy (IMRT) for lung tumors $[9,10]$. Compared to three-dimensional conformal radiotherapy (3D-CRT), IMRT planning using SPECT data could enhance the sparing of normal tissue and reduce the MLD [11]. Step-and-shoot and sliding-window IMRTs, the currently used IMRT techniques, can be used for inverse planning only after the number of beams and beam angles have been manually set. Studies on the quality of IMRTs with different beam arrangements have yielded inconsistent results [12, 13]. Many authors believe that using fewer beams may be beneficial in preserving perfusion of the normal lung $[14,15]$, a factor that has recently been implicated in acute radiation pneumonitis [16, 17]. Li-Jie Yin et al. found that PET-CT allows a better distinction between the collapsed lung tissue and tumor tissue, improving the accuracy of radiotherapy target delineation, and reducing radiation damage to the surrounding organs at risk (OARs) in nonsmall cell lung cancer (NSCLC) patients with atelectasis [18]. We used SPECT to evaluate lung perfusion before IMRT, and could direct the radiation away from functioning lung tissue and other organs such as the heart, spinal cord and esophagus, while maintaining target coverage in 10 randomly selected lung cancer patients. We also assessed whether the appropriate selection of the number of beams could further improve functional lung sparing while maintaining target coverage. 


\section{Material and methods}

\section{Patient population and ethical concerns}

The study protocol was approved by the ethics committee of our hospital. The study involved 10 patients who were scheduled to undergo radical radiotherapy for stage I-III non-small cell lung cancer (NSCLC), between December 2009 and October 2010. Six patients were men and four were women. Their median age was 61 years (range, 42-80 years).

\section{Imaging and image co-registration}

Computed tomography (CT; Biograph 16-PET/CT, Siemens, Erlangen, Germany) simulations were performed using 5-mm-thick axial slices, with the patients immobilized in a supine position with vacuum bags. CT image acquisition was performed using the following parameters: $120 \mathrm{kV}$ and $160 \mathrm{~mA}$. SPECT images depicting lung perfusion were obtained using a scanner equipped with double head detectors (Philips Forte, the Netherlands), following the intravenous administration of macro-agglomerated human serum albumin tagged with the radionuclide ${ }^{99 \mathrm{~m} T C}$ ( $99 \mathrm{mTC}$ MAA; Victor Medical Co. Ltd., Australia). The SPECT data were acquired using step-and-shoot motion at $6^{\circ}$ intervals on a $128 \times 128$ matrix (pixel size, $4.42 \mathrm{~mm}$ ) over $360^{\circ}$ (each head rotating through $180^{\circ}$ ). All scans were obtained during free breathing, and had sufficient coverage to include the total lung volume. Five points marked with metal crosses in the same place were used, and the CT and SPECT scans were co-registered manually in the Pinnacle3 planning system. Use lung perfusion fused imaging simulation CT plan. The entire lung was divided into functional and non-functional parts, and SPECT lung perfusion images were used to plan IMRT. The gross tumor volume (GTV) was contoured to include the primary tumor and lymph nodes that had a diameter $>1 \mathrm{~cm}$, and the planning target volume (PTV) was created by adding a uniform, $1-2 \mathrm{~cm}$ margin around the GTV. We planned to deliver a dose of 60 Gy to $95 \%$ of the PTV. The radiotherapy plans were assessed using dose-volume histograms (DVHs) with isodoses and conformity index $(\mathrm{Cl})$. The FV20 was $\leq 20 \%$. The volume of the spinal cord that received a dose of $<45 \mathrm{~Gy}$ and of the heart that received a dose < 40 Gy (V40) was less than 30\%.

\section{Radiotherapy planning}

In the current study, we administered step-and-shoot (static), fixed-field IMRT with a multileaf collimator integrated with an Elekta linear accelerator (Elekta Limited, Stockholm, Sweden). IMRT was planned using a Pinnacle 9.0 treatment planning system, with $6 \mathrm{MV}$ x-ray beams. Four types of IMRT plans, namely, the seven-beam CT (CT-7) plan, seven-beam SPECT (SPECT-7) plan, five-beam SPECT (SPECT-5) plan and four-beam SPECT (SPECT-4) plan, were generated with coplanar beams (Fig. 1-3). These plans differed in terms of beam angles and number of beams. In the CT-7 plan and the SPECT-7 plan, seven equally spaced beams were used, and the most beneficial beams for right lung tumors were those at $0^{\circ}, 40^{\circ}, 160^{\circ}, 200^{\circ}, 240^{\circ}, 280^{\circ}$ and $320^{\circ}$; for left lung tumors, the most beneficial beam directions were $0^{\circ}, 40^{\circ}$,

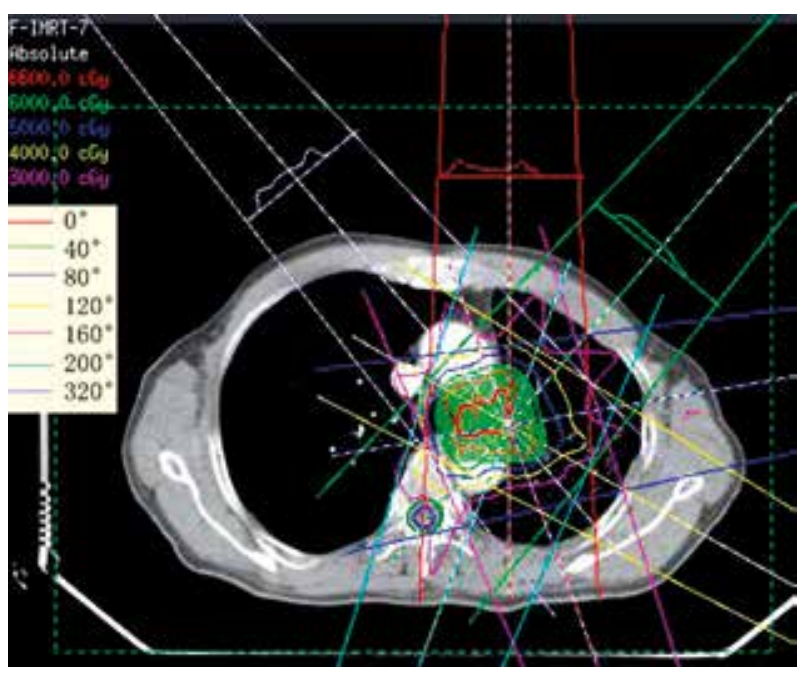

Fig. 1. Example of a seven-beam radiotherapy plan for a patient with left lung cancer

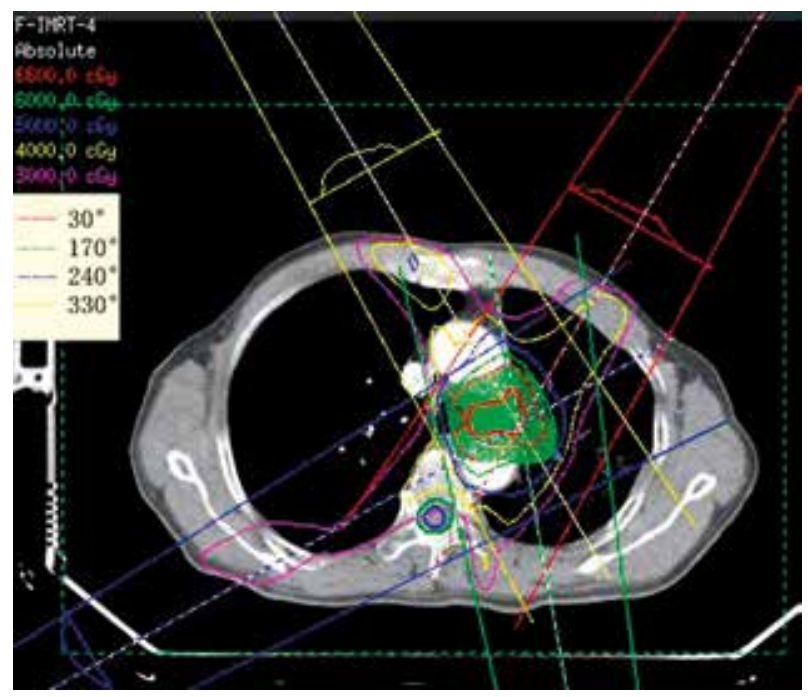

Fig. 2. Example of a four-beam radiotherapy plan for a patient with left lung cancer

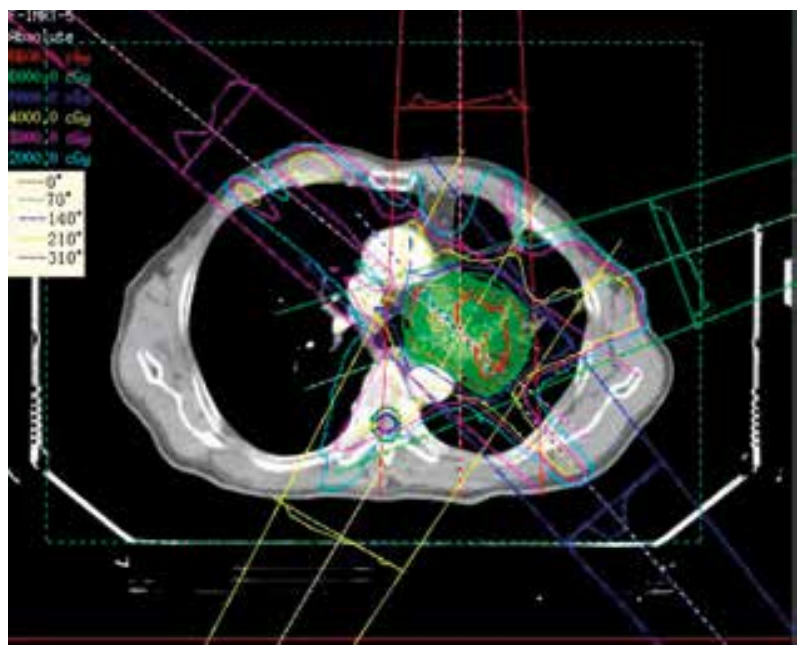

Fig. 3. Example of a five-beam radiotherapy plan for a patient with left lung cancer 
$80^{\circ}, 120^{\circ}, 160^{\circ}, 200^{\circ}$ and $320^{\circ}$. The SPECT-5 plan involved five equally spaced beams; beams angled at $0^{\circ}, 50^{\circ}, 150^{\circ}, 220^{\circ}$ and $290^{\circ}$ were used for right lung tumors, and those angled at $0^{\circ}, 70^{\circ}, 140^{\circ}, 210^{\circ}$ and $310^{\circ}$ were used for left lung tumors. The SPECT-4 plan used four equally spaced beams angled at $30^{\circ}, 160^{\circ}, 220^{\circ}$ and $330^{\circ}$ for right lung tumors and at $30^{\circ}$, $170^{\circ}, 240^{\circ}$ and $330^{\circ}$ for left lung tumors. The principal objectives of each plan were to minimize FV20 and dose variation within the PTV. The plans were prepared to administer $60 \mathrm{~Gy}$ in 30 fractions at the isocenter, using the $6 \mathrm{MV}$ X-ray beam data; $95 \%$ of the PTV received the prescribed dose. The doses to the functional lung and the esophagus, spinal cord and heart were minimized.

\section{Comparison of radiotherapy plans}

1. The prescribed dose of each IMRT plan for each patient was normalized to $60 \mathrm{~Gy}$, and the following parameters were compared between plans: Cl, FV20, FV30, MLD, maximum dose delivered to the spinal cord and $\mathrm{V} 40$ of the heart.

$$
\mathrm{Cl}=\left[\mathrm{V}_{\mathrm{PTV}(\geq p)} / \mathrm{V}_{\mathrm{PTV}}\right] \times\left[\mathrm{V}_{\mathrm{PTV}(\geq p)} / \mathrm{V}_{\mathrm{p}}\right]
$$

where $V_{\mathrm{PTV}(\geq \mathrm{p})}$ is the volume receiving $\geq \mathrm{x} \%$ of the prescribed dose, $V_{\text {PTV }}$ is equal to the PTV and $V_{p}$ is the coverage volume of the prescribed isodose line. The $\mathrm{Cl}$ represents the dose fit of the PTV relative to the volume covered by the prescribed isodose line. The closer the value of $\mathrm{Cl}$ is to 1 , the better the conformity of the PTV.

2. Target dose heterogeneity was assessed using a heterogeneity index (HI), as defined below:

$$
H I=D_{\max } / D_{\min }
$$

Table 1. Functional Lung volume receiving $\geq 20$ Gy (FV20) as a percentage of the total functional lung volume for the seven-beam computed tomography (CT-7) plan and the seven-beam single-photon emission CT (SPECT-7) plan

\begin{tabular}{|ccccc|}
\hline Patient & CT-7 (\%) & SPECT-7 (\%) & Change (\%) & \% Reduction* \\
\hline 1 & 7.64 & 6.26 & 1.38 & 18.06 \\
\hline 2 & 6.6 & 5.32 & 1.28 & 19.39 \\
\hline 3 & 14.86 & 7.91 & 6.95 & 46.77 \\
\hline 4 & 12.44 & 12.51 & -0.07 & -0.56 \\
\hline 5 & 19 & 13.69 & 5.31 & 27.95 \\
\hline 6 & 18.28 & 14.06 & 4.22 & 23.09 \\
\hline 7 & 8.61 & 7.55 & 1.06 & 12.31 \\
\hline 8 & 13.33 & 6.98 & 6.35 & 47.64 \\
\hline 9 & 6.63 & 5.02 & 1.61 & 24.28 \\
\hline 10 & 8.07 & 4.74 & 3.33 & 41.26 \\
\hline & Average & & $3.14 \pm 2.46$ & $26.02 \pm 15.45$ \\
\hline
\end{tabular}

*Reduction in FV2O in the SPECT-7 plan as compared to FV2O in the CT-7 plan where $D_{\max }$ is the largest dose to the target coverage, and $D_{\min }$ is the smallest dose to the target coverage. The smaller the value of $\mathrm{HI}$, the better the heterogeneity of the target coverage.

\section{Statistical analysis}

Statistical analyses were performed using the Statistical Package for the Social Sciences (SPSS) 13.0 (SPSS, Chicago, IL). All data were analyzed using means and standard deviations. Parameters were compared between plans using a paired $t$-test. A $p$ value $<0.05$ (two-tailed) was considered statistically significant.

\section{Results}

The FV2O and FV30 values and the corresponding reductions obtained using the SPECT-7 plan versus the CT-7 plan in all patients are shown in Tables 1 and 2. In most patients, the values were better in the SPECT-7 plan than in the CT-7 plan, with an average reduction in FV2O and FV30 of $26.02 \% \pm 15.45 \%$ and $14.41 \% \pm 16.66 \%$, respectively. In patients 6 and 7, however, the FV30 was higher in the SPECT-7 plan than in the CT-7 plan.

\section{Lung tissue dose}

Dose-volume histograms of different plans are shown in Figs. 4 and 5. The lung doses and other radiotherapy parameters are shown in Table 3. The FV5 value for the lung was highest in the SPECT-7 plan. The FV2O and FV30 values were lowest in the SPECT-7 plan. Thus, the SPECT-4 plan may be beneficial in lowering lung volumes receiving low-dose radiation, while the SPECT-7 plan may be beneficial in lowering lung volumes receiving high-dose radiation. The MLD was lower in the SPECT-4 plan than in the

Table 2. Functional Lung volume receiving $\geq 30$ Gy (FV30) as a percentage of the total functional lung volume for the seven-beam computed tomography (CT-7) Plan and the seven-beam single-photon emission CT (SPECT-7) plan

\begin{tabular}{|ccccc|}
\hline Patient & CT-7 (\%) & SPECT-7 (\%) & Change (\%) & \% Reduction* \\
\hline 1 & 2.41 & 2.2 & 0.21 & 8.71 \\
\hline 2 & 3.89 & 2.56 & 1.33 & 34.19 \\
\hline 3 & 3.31 & 3.11 & 0.2 & 6.04 \\
\hline 4 & 7.07 & 6.62 & 0.45 & 6.37 \\
\hline 5 & 8.64 & 6.28 & 2.36 & 27.32 \\
\hline 6 & 4.89 & 5.63 & -0.74 & -15.13 \\
7 & 3.19 & 3.23 & -0.04 & -1.25 \\
\hline 8 & 6.5 & 4.88 & 1.62 & 24.92 \\
\hline 9 & 3.23 & 2.74 & 0.49 & 15.17 \\
10 & 3.02 & 1.88 & 1.14 & 37.75 \\
& Average & & $0.70 \pm 0.91$ & $14.41 \pm 16.66$ \\
\hline
\end{tabular}

*Reduction in FV3O in the SPECT-7 plan as compared to FV3O in the CT-7 plan 


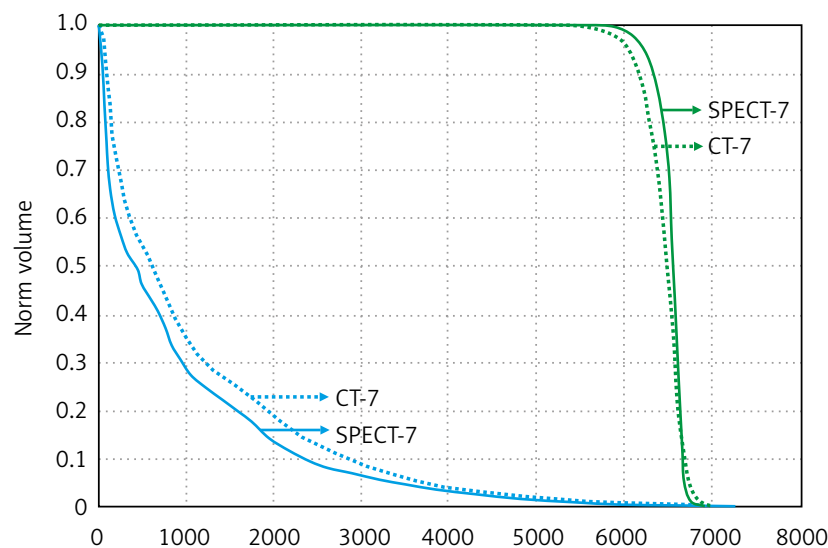

Fig. 4. Dose-volume histograms of seven-beam computed tomography (CT-7) and single-photon emission CT (SPECT-7) plans for the planning target volume (PTV; green lines) and the total lung volume (blue lines) of a patient with stage III non-small cell lung cancer

SPECT-5 and SPECT-7 plans; the MLD did not significantly differ between the SPECT-5 and SPECT-7 plans.

\section{Radiation doses in other organs}

The radiation doses in other organs are shown in Table 3. The $\mathbf{V} 40$ for the heart and the maximum dose in the spinal cord did not significantly differ among the different plans.

\section{Conformity index of planing target volume}

The $\mathrm{CI}$ significantly differed between the SPECT-7 and SPECT-4 plans and between the SPECT-5 and SPECT-4 plans, but not between the SPECT-5 and SPECT-7 plans. The conformity of the SPECT-5 and SPECT-7 plans was better than that of the SPECT-4 plan.

\section{Discussion}

In 1995, Marks et al. [19] first used SPECT to plan radiotherapy in order to reduce lung radioactive damage. They divided the lung into functional and non-functional parts on the basis of lung perfusion and formulated radiotherapy plans to minimize incidental irradiation of the functioning tissue. A SPECT-guided 3D-CRT plan reduces FV20 and FV30 values to a greater extent than conventional 3D-CRT [20, 21].

At present, IMRT technology is being increasingly applied in lung cancer treatments. We integrated SPECT lung perfusion into IMRT planning, by optimizing beam direction, and explored the differences in various parameters among IMRT plans involving different beam directions. The aim was to improve the target dose while reducing damage to the normal lung tissue.

Liu et al. [22] found that in nine of ten patients, the FV5 for the lung was higher with fixed-field, dynamic IMRT than with 3D-CRT; however, this value could be reduced by decreasing the number of radiation beams. In addition to increasing the lung volume receiving low-dose irradiation, a large number of beams has been reported to directly increase the duration of treatment, thereby increasing patient discomfort during treatment and possibly affecting the bio-

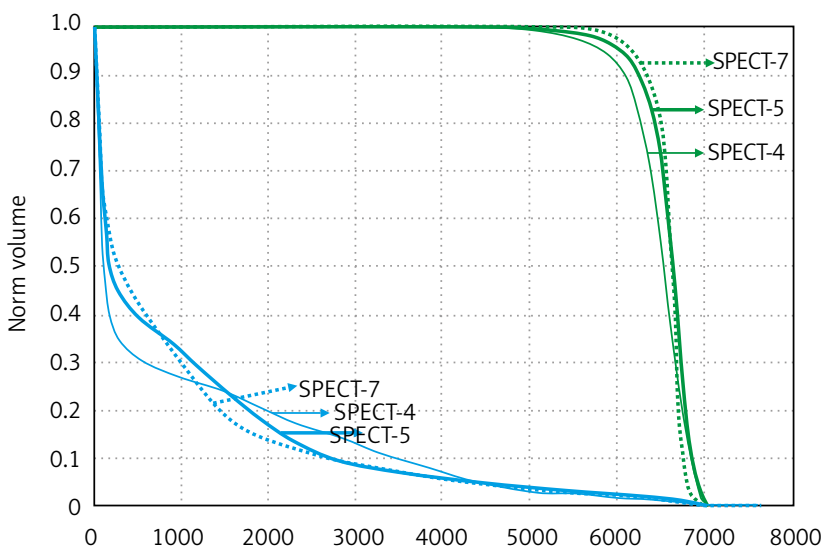

Fig. 5. Dose-volume histograms of four-, five- and seven-beam single-photon emission computed tomography (SPECT-4, SPECT-5 and SPECT-7, respectively) for the planning target volume (PTV; green lines) and total lung volume (blue lines) of a patient with stage III non-small cell lung cancer

Table 3. Comparison of dose-related parameters between different radiotherapy plans

$\begin{array}{lccc}\text { Parameter } & \text { SPECT-4 } & \text { SPECT-5 } & \text { SPECT-7 } \\ \text { FV5 (\%) } & 26.56 \pm 10.25^{\Delta 1} & 36.84 \pm 11.31^{\star 1} & 41.80 \pm 14.29^{\diamond 1} \\ \text { FV13 (\%) } & 17.52 \pm 6.81 & 18.40 \pm 6.26 & 18.06 \pm 10.27 \\ \text { FV20 (\%) } & 10.60 \pm 3.40 & 10.14 \pm 3.63^{\star 2} & 8.40 \pm 3.63^{\diamond 2} \\ \text { FV30 (\%) } & 5.61 \pm 2.38^{\Delta 2} & 4.39 \pm 1.95^{\star 3} & 3.91 \pm 1.77^{\diamond 3} \\ \text { MLD (Gy) } & 9.02 \pm 2.55^{\Delta 3} & 9.55 \pm 2.63 & 9.64 \pm 2.87^{\diamond 4} \\ \text { Cord max (Gy) } & 39.42 \pm 9.73 & 39.32 \pm 8.66 & 38.51 \pm 7.09 \\ \text { Heart V } \text { (\%) } & 6.47 \pm 10.24 & 7.04 \pm 11.55 & 5.85 \pm 10.19 \\ \text { Cl } & 0.77 \pm 0.61^{\triangle 4} & 0.86 \pm 0.03 & 0.87 \pm 0.05^{\diamond 5} \\ \text { HI } & 1.74 \pm 0.33 & 1.56 \pm 0.24^{\star 4} & 1.44 \pm 0.11\end{array}$

All values are expressed as mean and standard deviation.

${ }^{4}$ Compared to the five-beam single-photon emission computed tomography (SPECT-5) plan: $11,2,3,4=-4.403,2.659,-3.422,4.307 ; p<0.05$.

"Compared to the seven-beam SPECT (SPECT-7) plan: $t 1,2,3,4=-3.087,3.681$, 3.408, 2.498; $p<0.05$.

Compared to the four-beam SPECT (SPECT-4) plan: t1,2,3,4,5 =-4.409, 2.322,

$3.433,-2.543,6.503,3.317, p<0.05$.

logical effects of the treatment [23]. Christian et al. [24] also compared 3D-CRT plans and IMRT plans with different beam arrangements. They used a series of three-, five-, seven- and nine-beam coplanar beam arrangements as well as one sixbeam non-coplanar arrangement. All of the IMRT plans, except for the three-beam arrangement, improved the ratio of PTV coverage to lung FV20 values over the 3D-CRT plans; the nine-beam arrangement was best. However, the DVH of the best nine-beam IMRT plan showed an increase in the lung volume receiving low-dose radiation, when compared to the 3D-CRT plan [24].

Many recent studies exploring automatic, beam-angle optimization have reported that the quality of IMRT plans can be maintained or even improved by optimizing beam direction and reducing the number of beams. Liu et al. [25] designed and used an automatic, beam-angle optimization technique in 10 patients with NSCLC; they found that the quality of SPECT-5 was not inferior to that of SPECT-7 or SPECT-9. Taiki Magome et al. [26] suggested that a comput- 
er-aided method for determination of beam arrangements based on similar cases in a radiotherapy treatment-planning database for stereotactic lung radiation therapy may provide usable beam arrangements, which have no statistically significant differences.

Soderstrom et al. [27] suggested that improvement of the conformity of the target played a negative role in protecting normal tissues when more than nine beams were used for IMRT in patients with NSCLC. They recommend that fewer than nine beams be used for IMRT. McGuire et al. [28] compared nine-beam CT, nine-beam SPECT and SPECT-4 plans in terms of parameters such as FV20 and FV30, in five patients with lung cancer who underwent lung perfusion SPECT and dynamic, fixed-field IMRT. The results showed that low doses delivered to the functional lung were reduced only in the case of the four-beam plan, which was advantageous for lung protection. This suggests that using fewer beams to plan IMRT for lung cancer may be beneficial for reducing both the acute and chronic complications of radiotherapy.

We analyzed the advantages of SPECT-guided IMRT in reducing the dose delivered to the functional lung and explored the effects of different beams on IMRT planned using SPECT lung perfusion. We found that FV20 and FV30 decreased by $26.02 \% \pm 15.45 \%$ and $14.41 \% \pm 16.66 \%$, respectively, in the SPECT-7 plan as compared with the CT-7 plan. We also found that compared with the SPECT-7 and SPECT-5 plans, the SPECT-4 plan helped to minimize lowdose radiation to the normal lung and the MLD, whereas the SPECT-7 plan was superior to the SPECT-4 and SPECT-5 plans in terms of minimizing high-dose radiation to the normal lung and improving $\mathrm{Cl}$ and $\mathrm{HI}$. The radiation doses to other vital organs near the lung did not significantly differ between plans. The mechanism underlying radiation-induced pneumonitis is not fully understood, but evidence shows that the acute effects are caused by the delivery of low doses to a large lung volume. Novakova-Jiresova et al. [29] found in an animal model that the delivery of high radiation doses to small volumes is less damaging to overall lung function than the delivery of low doses to large volumes. Wang et al. [30] found that patients in whom a relatively large lung volume had received a dose of 5 Gy developed pulmonary complications. Allen et al. [31] also found that the FV5 value was an important factor in patients who developed pneumonitis after IMRT. Predictive modeling concurs with these clinical findings and suggests that low-dose radiation contributes to radiation pneumonitis. If the above hypothesis is correct, then the SPECT-4 plan may reduce the lung volume receiving low-dose radiation and, therefore, pulmonary complications, while providing satisfactory target coverage.

In conclusion, this study showed that the incorporation of SPECT images into IMRT planning may help reduce the amount of dose delivered to the functional lung. The use of SPECT-guided plans may reduce the incidence of pneumonitis by reducing the volume of the functional lung receiving doses of at least 20 or 30 Gy. The number of radiation beams influenced the dose distribution to the lung cancer during IMRT. Reduction in the number of beams helped reduce the MLD and the incidence of radiation pneumo- nitis, whereas increase in the number of beams improved the $\mathrm{Cl}$ without reducing the radiation dose to normal tissue. Therefore, each of these beam arrangements should be considered to plan the optimal IMRT in clinical practice. Further studies with a greater number of patients are required, since the variability between patient anatomy and functional lung maps is a considerable factor in SPECT-guided treatment planning.

The authors declare no conflict of interest.

\section{References}

1. Parkin DM, Bray F, Ferlay J, Pisani P. Global cancer statistics, 2002. CA: Cancer J Clin 2005; 55: 74-108.

2. Kozielski J, Kaczmarczyk G, Porębska I, Szmygin-Milanowska K, Gołecki M. Lung cancer in patients under the age of 40 years. Contemp Oncol (Pozn) 2012; 16: 413-5.

3. Langfort R, Szołkowska M, Szczepulska-Wójcik E, Qandil N, Maksymiuk B, Giedronowicz D, Rudziński P, Orłowski TM. The importance of immunohistochemistry for differentiation of lung adenocarcinoma and squamous cell carcinoma in small samples and cytology specimens. Kardiochirur Torakochirur Pol 2012; 9: 202-208.

4. Arriagada R, Komaki R, Cox JD. Radiation dose escalation in nonsmall cell carcinoma of the lung. Semin Radiat Oncol 2004; 14 287-91.

5. Graham MV, Purdy JA, Emami B, Harms W, Bosch W, Lockett MA, Perez CA. Clinical dose-volume histogram analysis for pneumonitis after 3d treatment for non-small cell lung cancer (NSCLC). Int J Radiat Oncol Biol Phys 1999; 45: 323-9.

6. Kwa SL, Lebesque JV, Theuws JC, Marks LB, Munley MT, Bentel G, Oetzel D, Spahn U, Graham MV, Drzymala RE, Purdy JA, Lichter AS, Martel MK, Ten Haken RK. Radiation pneumonitis as a function of mean lung dose: An analysis of pooled data of 540 patients. Int J Radiat Oncol Biol Phys 1998; 42: 1-9.

7. Seppenwoolde Y, Lebesque JV, de Jaeger K, Belderbos JS, Boersma LJ, Schilstra C, Henning GT, Hayman JA, Martel MK, Ten Haken RK. Comparing different ntcp models that predict the incidence of radiation pneumonitis. Normal tissue complication probability. Int J Radiat Oncol Biol Phys 2003; 55: 724-35.

8. Seco J, Panahandeh HR, Westover K, Adams J, Willers H. Treatment of non-small cell lung cancer patients with proton beam-based stereotactic body radiotherapy: dosimetric comparison with photon plans highlights importance of range uncertainty. Int J Radiat Oncol Biol Phys 2012; 83: 354-361.

9. Christian JA, Partridge M, Nioutsikou E, Cook G, McNair HA, Cronin $B$, Courbon F, Bedford JL, Brada M. The incorporation of spect functional lung imaging into inverse radiotherapy planning for nonsmall cell lung cancer. Radiother Oncol 2005; 77: 271-7.

10. Munley MT, Marks LB, Scarfone C, Sibley GS, Patz EF Jr, Turkington TG, Jaszczak RJ, Gilland DR, Anscher MS, Coleman RE. Multimodality nuclear medicine imaging in three-dimensional radiation treatment planning for lung cancer: Challenges and prospects. Lung Cancer 1999; 23: 105-14.

11. Lavrenkov K, Christian JA, Partridge M, Niotsikou E, Cook G, Parker M, Bedford JL, Brada M. A potential to reduce pulmonary toxicity: The use of perfusion spect with imrt for functional lung avoidance in radiotherapy of non-small cell lung cancer. Radiother Oncol 2007; 83: 156-62.

12. ZHU Zheng-fei, XU Zhi-yong, CHEN Lan-fei, HU Wei-gang, FAN Min, WU Kai-liang, XIA Bing, FU Xiao-long. Impact of different beam set-up methods on quality of intensity modulated radiation therapy in nonsmall cell lung cancer. Chin J Radiol Med Prot 2010; 30: 1.

13. Lim do H, Yi BY, Mirmiran A, Dhople A, Suntharalingam M, D'Souza WD. Optimal beam arrangement for stereotactic body radiation therapy delivery in lung tumors. Acta Oncol 2010; 49: 219-24. 
14. Choi Y, Kim JK, Lee HS, Hur WJ, Chai GY, Kang KM. Impact of intensity-modulated radiation therapy as a boost treatment on the lung-dose distributions for non-small-cell lung cancer. Int J Radiat Oncol Biol Phys 2005; 63: 683-9.

15. Yom SS, Liao Z, Liu HH, Tucker SL, Hu CS, Wei X, Wang X, Wang S, Mohan R, Cox JD, Komaki R. Initial evaluation of treatment-related pneumonitis in advanced-stage non-small-cell lung cancer patients treated with concurrent chemotherapy and intensity-modulated radiotherapy. Int J Radiat Oncol Biol Phys. 2007; 68: 94-102.

16. Yorke ED, Jackson A, Rosenzweig KE, Braban L, Leibel SA, Ling CC. Correlation of dosimetric factors and radiation pneumonitis for non-small-cell lung cancer patients in a recently completed dose escalation study. Int J Radiat Oncol Biol Phys 2005; 63: 672-82.

17. Wang S, Liao Z, Wei X, Liu HH, Tucker SL, Hu CS, Mohan R, Cox JD, Komaki R. Analysis of clinical and dosimetric factors associated with treatment-related pneumonitis (TRP) in patients with nonsmall-cell lung cancer (NSCLC) treated with concurrent chemotherapy and three-dimensional conformal radiotherapy (3D-CRT). Int J Radiat Oncol Biol Phys 2006; 66: 1399-407.

18. Yin LJ, Yu XB, Ren YG, Gu GH, Ding TG, Lu Z. Utilization of PET-CT in target volume delineation for three-dimensional conformal radiotherapy in patients with non-small cell lung cancer and atelectasis. Multidiscip Respir Med. 2013; 8: 21.

19. Marks LB, Spencer DP, Sherouse GW, Bentel G, Clough R, Vann K, Jaszczak R, Coleman RE, Prosnitz LR. The role of three dimensional functional lung imaging in radiation treatment planning: The functional dose-volume histogram. Int J Radiat Oncol Biol Phys 1995; 33: 65-75.

20. Seppenwoolde Y, Muller SH, Theuws JC, Baas P, Belderbos JS, Boersma LJ, Lebesque JV. Radiation dose-effect relations and local recovery in perfusion for patients with non-small-cell lung cancer. Int J Radiat Oncol Biol Phys 2000; 47: 681-90.

21. McGuire SM, Zhou S, Marks LB, Dewhirst M, Yin FF, Das SK. A methodology for using spect to reduce intensity-modulated radiation therapy (IMRT) dose to functioning lung. Int J Radiat Oncol Biol Phys 2006; 66: 1543-52.

22. Liu HH, Wang X, Dong L, Wu Q, Liao Z, Stevens CW, Guerrero TM, Komaki R, Cox JD, Mohan R. Feasibility of sparing lung and oth er thoracic structures with intensity-modulated radiotherapy for non-small-cell lung cancer. Int J Radiat Oncol Biol Phys 2004; 58: 1268-79.

23. Fowler JF, Welsh JS, Howard SP. Loss of biological effect in prolonged fraction delivery. Int J Radiat Oncol Biol Phys 2004; 59: 242-9.

24. Christian JA, Bedford JL, Webb S, Brada M. Comparison of inverse-planned three-dimensional conformal radiotherapy and intensity-modulated radiotherapy for non-small-cell lung cancer. Int J Radiat Oncol Biol Phys 2007; 67: 735-41.

25. Liu HH, Jauregui M, Zhang X, Wang X, Dong L, Mohan R. Beam angle optimization and reduction for intensity-modulated radiation therapy of non-small-cell lung cancers Int J Radiat Oncol Biol Phys 2006 ; 65: 561-72.

26. Magome T, Arimura H, Shioyama Y, Mizoguchi A,Tokunaga C, Nakamura K, Honda H, Ohki M, Toyofuku F, Hirata H. Computer-aid ed beam arrangement based on similar cases in radiation treatment-planning databases for stereotactic lung radiation therapy. J Radiat Res 2013; 54: 569-77.

27. Söderström S, Brahme A. Which is the most suitable number of photon beam portals in coplanar radiation therapy? Int J Radiat Oncol Biol Phys 1995; 33:151-9.

28. McGuire SM, Marks LB, Yin FF, Das SK. A methodology for selecting the beam arrangement to reduce the intensity-modulated radiation therapy (IMRT) dose to the spect-defined functioning lung. Phys Med Biol 2010; 55: 403-16

29. Novakova-Jiresova A, van Luijk P, van Goor H, Kampinga HH, Cop pes RP. Changes in expression of injury after irradiation of in creasing volumes in rat lung. Int I Radiat Oncol Biol Phys 2007; 67: 1510-8

30. Wang SL, Liao Z, Vaporciyan AA, Tucker SL, Liu H, Wei X, Swisher S, Ajani JA, Cox JD, Komaki R. Investigation of clinical and dosimetric factors associated with postoperative pulmonary complications in esophageal cancer patients treated with concurrent chemora- diotherapy followed by surgery. Int J Radiat Oncol Biol Phys 2006; 64: 692-9.

31. Allen AM, Czerminska M, Janne PA, Sugarbaker DJ, Bueno R, Harris JR, Court L, Baldini EH. Fatal pneumonitis associated with intensity-modulated radiation therapy for mesothelioma. Int J Radiat Oncol Biol Phys 2006; 65: 640-5.

\section{Address for correspondence}

\section{Yanming Wang}

Department of Radiotherapy

The General Hospital of Jinan Military Command

25 Shifan Road

250031 Jinan, China

tel. (86531) 51666640

e-mail: ymwang64@hotmail.com

Submitted: 1.12 .2013

Accepted: $\quad 3.02 .2014$ 